\title{
A case report of urethral prolapse in a 38 year old female with 46XY karyotype
}

\author{
Helena Watson, Ewa Stasiowska \\ University Hospital Lewisham, London, UK.
}

\begin{abstract}
Summary A 38-year old female presented with the acute onset of a vulval mass associated with pain and vaginal bleeding. She is female phenotype but has 46XY karyotype and Complete Androgen Insensitivity Syndrome (CAIS). At 15 years old she had a laparotomy and bilateral orchidectomy. Following admission, an examination under anaesthesia and cystoscopy was performed. A diagnosis of strangulated complete urethral prolapse was made. The lesion was excised with diathermy and the meatal skin was reanastomosed to the urethra. At follow-up, the urethra was well healed. The patient now attends Menopause Clinic for oestrogen-replacement therapy. We hope this case raises awareness of the possibility of urethral prolapse in younger women who are oestrogen deficient. It provides further incentive for compliance with hormonereplacement therapy for patients with CAIS following gonadectomy, or other women with premature menopause.
\end{abstract}

KEY WORDS: Complete urethral prolapse; Complete Androgen Insensitivity Syndrome; Oestrogen deficiency.

Submitted 22 April 2014; Accepted 31 May 2014

\section{Case report}

A 38-year old female was referred to a district general hospital in South London. She presented with the acute onset of a vulval mass over a few days, associated with pain and vaginal bleeding. She had dysuria and urinary frequency, but no voiding problems. She is female phenotype but has 46XY karyotype and complete androgen insensitivity syndrome (CAIS). Her family history includes an aunt and cousin who had CAIS. At 15 years old she had a laparotomy and bilateral orchidectomy. She was commenced on hormone-replacement therapy following her surgery but had been non-compliant for a few years before presentation.

Following admission, an examination under anaesthesia and cystoscopy was performed. An erythematous, cyanotic, doughnut-shaped mass was protruding from the anterior vaginal wall containing distal urethral mucosa. The bladder and proximal urethra appeared normal.
She had a normal length vagina with a well-supported vaginal vault. A diagnosis of strangulated complete urethral prolapse was made. The lesion was excised with diathermy, the meatal skin was re-anastomosed to the urethra and a vaginal pack and in-dwelling catheter were inserted. The pack was removed the next day and the patient discharged to return in one week for review and a trial without catheter. At follow-up, the urethra was well healed. The patient now attends menopause clinic for oestrogen-replacement therapy.

\section{Discussion}

We present this case because of its rarity among women in this age group and because of its connection to the individual's genetic status and her oestrogen deficiency. Urehral prolapse is defined as the complete eversion of the terminal urethra through the external urethral meatus. It is an uncommon condition usually seen in prepubertal girls and postmenopausal women. Approximately $80 \%$ of cases are in the paediatric population where the incidence is $1: 3000$ (1). There is no reference in the literature to cases amongst women in their 30s, nor in the Androgen Insensitivity Syndrome population.

The urethra is composed of inner longitudinal and outer circular-oblique smooth muscle layers. It is a disruption in the natural cleavage plane between these two layers, which results in eversion of the urethral mucosa through the meatus. Thus, although the precise cause of urethral prolapse is unknown, likely causes are congenital or acquired defects of the urethra, in particular where there is a weakened attachment between the two muscle layers. Congenital defects include abnormally wide or patulous urethra, neuromuscular disorders or abnormal elastic tissue. Acquired causes include trauma from childbirth, and rarely periurethral bulking agents (1). A further acquired risk factor is oestrogen deficiency: the mucosa and submucosal vascular plexus are both responsive to oestrogen, when they become atrophied the mucosal seal may be lost, predisposing to stress incontinence and urethral prolapse.

Oestrogen deficiency certainly seems to have played a role in our case, in light of the patient's CAIS. In CAIS there are no visible signs of androgen action and the subjects are born with normal female external genitalia. The diag- 
nostic criteria also include male (46XY) karyotype, presence of testes, normal testosterone production, absence of Mullerian duct remnants and spontaneous feminisation at puberty with no virilisation (1). In CAIS it is the usual practice to perform a gonadectomy due to an increased risk of testicular malignancies in these patients. In Nakhal et al. retrospective evaluation of retained testes in adolescents with CAIS, pre-malignant foci were found in three out of 25 patients (1). After gonadectomy, oestrogen-only hormone replacement therapy is commenced to prevent menopausal symptoms and osteoporosis. The correlation of non-compliance with oestrogen therapy and presentation of urethral prolapse, suggests that oestrogen deficiency was a contributory cause.

Another possibility is that given $5 \alpha$-dihydrotestosterone's role in embryological development of the urogenital sinus, our patient has some subtle anatomical variation that contributed to urethral prolapse. The phenotypic variations in partial androgen insensitivity syndrome range from hypospadias in male genitalia to female genitalia with a blind-ending vaginal pouch. However there are no urological abdormalities described in the literature for CAIS, and if an anatomical variation in the urethra was significant, we might have expected it to cause paediatric urethral prolapse.

The clinical presentation of urethral prolapse depends on the age group. In the paediatric population it is typically asymptomatic, but occasionally urinary frequency, dysuria or pain are present. Postmenopausal women are usually symptomatic with vaginal bleeding, dysuria, urinary frequency and/or haematuria. On examination, urethral prolapse appears as an erythematous doughnutshaped mass protruding form the anterior vaginal wall. It may be infected, ulcerated or necrotic depending on the degree of ischaemia. Confirmation that the central opening of this mass is the urethral meatus, either by observation of micturition, catheterisation or cystoscopy, is diagnostic of urethral prolapse. Occasionally the diagnosis may only be confirmed on histology after excision of the mass.

Regarding management of urethral prolapse there are no randomised controlled trials comparing medical with surgical treatment and the majority of the experience reported in the literature is in the paediatric population. Traditionally urethral prolapse was treated surgically with excision or cautery. More recently medical treatments have become more popular. Rudin et al. experience with 58 cases, treated 20 medically, of which 5 had recurrence, 2 ultimately requiring surgery. In our case, the urethral mucosa was strangulated and ischaemic on presentation, necessitating surgical excision. However, if she had presented earlier topical oestrogens may well have been suitable, particularly in light of her hypo-oestrogenic state.

We hope this case raises awareness of the possibility of urethral prolapse in younger women who are oestrogen deficient. It provides further incentive for compliance with hormone-replacement therapy for patients with CAIS following gonadectomy, or other women with premature menopause.

\section{Consent}

The patient's consent was acquired for publication, under the understanding that her name, initials or hospital number, would not be published and all attempts made to ensure anonymity. The patient understood that material may be published in print and/or online, but that material would not be used for advertising or out of context. The signed consent form is retained by the corresponding author.

\section{REFERENCES}

1. Agzarian AE \& Agzarian AY. Urethral Prolapse, Report of a Case, Proceedings of UCLA Healthcare 2010; Vol. 14.

2. Harris RL, Cundiff GW, Coates KW, et al. Urethral Prolapse after collagen injection, AJOG. 1998; 178:P614-615.

3. Jaaskelainen J, Hughes IA. Androgen Insensitivity Sundromes, In Balen AH (ed.) Paediatric and Adolescent Gyanecology: A Multidisciplinary Approach, Cambridge University Press, Cambridge 2004, pp 253-263.

4. Nakhal RS, Hall-Craggs M, Freeman A, et al. Evaluation of retained testes in adolescent girls and women with complete androgen insensitivity syndrome, Radiology. 2013; 268:153-60.

5. Rudin JE, Geldt VG, Evgeny B. A Prolapse of urethral mucosa in white female children: experience with 58 cases, J Ped Surg. 1997; 32:423-525.

\section{Correspondence}

Dr Helena Watson, MB BChir MRCOG (Corresponding Author)

helenawatson85@gmail.com

Miss Ewa Stasiowska, MD PhD MRCOG

University Hospital Lewisham, Lewisham High Street, SE13 6LH,

London, UK 